\title{
VENTILATORY FUNCTION STUDIES IN EXTRAPERIOSTEAL PLOMBAGE
}

\author{
BY \\ H. MILWIDSKY AND H. ROMANOFF \\ From the Department of Thoracic Surgery, Hadassah University Hospital, Jerusalem, Israel
}

(RECEIVED FOR PUBLICATION MAY 7, 1955)

Classical thoracoplasty, being the recognized standard procedure for permanent collapse therapy in pulmonary tuberculosis, has yet to be surpassed by any other surgical method with regard to longterm arrest of the disease and sputum conversion. However, it is a multiple-stage procedure, requiring extensive decostalization followed by permanent deformity and considerable impairment of ventilatory function (Kaltreider, Fray, and Phillips, 1938; Birath, 1944; Powers and Himmelstein, 1951).

Extraperiosteal plombage procedures using lucite spheres (Wilson, 1946; Woods, Walker, and Schmidt, 1950 ; Woods and Buente, 1953) and polyethylene sponge (Engberg and Hansen, 1953) have been employed during recent years with very satisfactory results and appear to be relatively simple and safe substitutes for the classical multiple-stage thoracoplasty (Alexander, 1937). In addition, extraperiosteal plombage, by virtue of its negligible operative risk and low morbidity, can be applied to advanced bilateral cases where conventional thoracoplasty would involve too great a risk. In this procedure the requisite collapse is effected in a single stage with no paradoxical motion of the chest wall, and if the plombage material is not removed in a second stage no deformity whatsoever ensues.

Reports on pulmonary function studies in patients undergoing extraperiosteal plombage are few. The loss of ventilatory function appears to be much lower than after classical thoracoplasty (Watson and Gaensler, 1952). We therefore report the results of ventilatory function studies performed in 17 patients before and after extraperiosteal plombage. The post-operative tests were performed between two and six months after operation.

The 17 patients, whose clinical data are listed in Table I, represent only those plombage cases in which accurate ventilatory function tests could be obtained before and after operation. More detailed information and follow-up reports on our entire series of plombage thoracoplasties will be given in a separate article. In 11 patients, lucite spheres were used as plombage material. In eight of them, the plombe was removed in a second stage approximately three months after its insertion, together with resection of the deperiostealized rib segments. In three patients (Nos. 9, 10, 11) the lucite spheres were left as a permanent plombe. In the remaining six patients polyethylene sponges ("polystan") were used as a permanent plombe, and in three of them (Nos. 14, 15, 16) bilateral plombage was done.

\section{Methods}

Ventilatory function tests were performed according to the method of Baldwin, Cournand, and Richards (1948) with a 9-litre Collins spirometer. The patient was in the supine position, breathing through a mouthpiece into a tank filled with oxygen and room air. After several ordinary breathings vital capacity was obtained by recording the amplitude of a forceful expiration following maximal inspiration. The maximum breathing capacity was recorded by urging the patient to breathe as rapidly and deeply as possible for 15 seconds. The values were calculated in litres per minute. The predicted amounts of vital capacity and maximum breathing capacity as well as the breathing reserve ratios were estimated according to the formulae of Baldwin and others (1948). The air velocity index was determined according to Gaensler (1950).

\section{Results}

The pre-operative vital capacities varied between 30 and $84 \%$ of the predicted, the average being $67 \%$. The pre-operative maximum breathing capacities values were between 23 and $83 \%$, the average being $50 \%$. The ventilation calculated in litres per minute showed pre-operative values ranging from 2.7 to $161 . / \mathrm{m}$. Eight patients showed definitely increased values indicating hyperventilation. The breathing reserve ratio ranged between 68 and $95 \%$, the average being $84.6 \%$, a definitely subnormal value (Cournand cited by Hochberg, 1952). The air velocity index ranged from 0.4 to $1.3 \%$. 
TABLE I

PRE- AND POST-OPERATIVE PULMONARY FUNCTION TESTS IN 17 CASES OF EXTRAPERIOSTEAL PLOMBAGE

\begin{tabular}{|c|c|c|c|c|c|c|c|c|c|c|c|c|c|}
\hline \multirow[b]{2}{*}{ No. } & \multirow[b]{2}{*}{ Patient } & \multirow[b]{2}{*}{ Age } & \multirow[b]{2}{*}{ Sex } & \multirow[b]{2}{*}{$\begin{array}{l}\text { Pulmonary } \\
\text { Tuberculosis }\end{array}$} & \multirow[b]{2}{*}{$\begin{array}{c}\text { Extraperiosteal } \\
\text { Plombage }\end{array}$} & \multirow[b]{2}{*}{ Clinical Result } & \multicolumn{5}{|c|}{ Pulmonary Function Tests } & \multicolumn{2}{|c|}{ Change $(\%)$} \\
\hline & & & & & & & $\begin{array}{l}\text { Vital } \\
\text { Capacity } \\
\text { (\% of } \\
\text { Predicted } \\
\text { Values) }\end{array}$ & \begin{tabular}{|c|} 
Maxi- \\
mum \\
Brea- \\
thing \\
Capa- \\
city (\% \\
of Pre- \\
dicted \\
values;
\end{tabular} & $\begin{array}{l}\text { Venti- } \\
\text { latory } \\
\text { Capa- } \\
\text { city } \\
(1 . \mathrm{m} .)\end{array}$ & $\begin{array}{c}\text { Brea- } \\
\text { thing } \\
\text { Re- } \\
\text { serve } \\
\text { Ratio }\end{array}$ & $\begin{array}{c}\text { Air } \\
\text { Velo- } \\
\text { city } \\
\text { Index }\end{array}$ & $\begin{array}{l}\text { Vital } \\
\text { Cap- } \\
\text { acity }\end{array}$ & $\begin{array}{l}\text { Maxi- } \\
\text { mum } \\
\text { Brea- } \\
\text { thing } \\
\text { Cap- } \\
\text { acity }\end{array}$ \\
\hline 1 & A. $\mathbf{S}$. & 19 & $\mathbf{M}$ & $\begin{array}{l}\text { Bilateral, advanced, } \\
\text { cavities R.U.L. } \\
\text { superior segment } \\
\text { R.L.L. }\end{array}$ & $\begin{array}{l}\text { Right, } 6 \text { ribs, lucite } \\
\text { balls, } 2 \text { stages }\end{array}$ & $\begin{array}{l}\text { Cavities closed, } \\
\text { sputum negative, } \\
\text { discharged }\end{array}$ & $\begin{array}{l}\text { Before op. } 82 \\
\text { After 2nd stage } 73\end{array}$ & $\begin{array}{l}46 \\
79\end{array}$ & $\begin{array}{l}7 \cdot 5 \\
4 \cdot 1\end{array}$ & $\begin{array}{l}87 \\
92\end{array}$ & $\begin{array}{r}0.5 \\
1.08\end{array}$ & -9 & +33 \\
\hline 2 & I. $\mathbf{H}$. & 30 & $\mathbf{M}$ & $\begin{array}{l}\text { Bilateral, advanced, } \\
\text { cavity L.U.L. }\end{array}$ & $\begin{array}{l}\text { Left, } 5 \text { ribs, lucite } \\
\text { balls, } 2 \text { stages }\end{array}$ & $\begin{array}{l}\text { Cavity closed, } \\
\text { sputum negative, } \\
\text { discharged }\end{array}$ & $\begin{array}{l}\text { Before op. } \\
\text { After 2nd stage } 63\end{array}$ & $\begin{array}{l}47 \\
71\end{array}$ & $\begin{array}{l}5.61 \\
3.93\end{array}$ & $\begin{array}{l}95 \\
92\end{array}$ & $\begin{array}{l}0.6 \\
1.13\end{array}$ & -15 & +24 \\
\hline 3 & S. K. & 39 & $\mathbf{M}$ & $\begin{array}{l}\text { Bilateral, far } \\
\text { advanced, } \\
\text { cavities L.U.L. }\end{array}$ & $\begin{array}{l}\text { Left, } 5 \text { ribs, lucite } \\
\text { balls, } 2 \text { stages }\end{array}$ & $\begin{array}{l}\text { Cavities closed, } \\
\text { sputum negative, } \\
\text { discharged }\end{array}$ & $\begin{array}{l}\text { Before op. } 77 \\
\text { After 2nd stage } 79\end{array}$ & $\begin{array}{l}40 \\
57\end{array}$ & $\begin{array}{l}6 \cdot 4 \\
5 \cdot 72\end{array}$ & $\begin{array}{l}85 \\
91\end{array}$ & $\begin{array}{l}0.52 \\
0.72\end{array}$ & +2 & +17 \\
\hline 4 & J. B. & 46 & $\mathbf{M}$ & $\begin{array}{l}\text { Bilateral, far } \\
\text { advanced, cavities } \\
\text { R.U.L. }\end{array}$ & $\begin{array}{l}\text { Right, } 6 \text { ribs, lucite } \\
\text { balls, } 2 \text { stages }\end{array}$ & $\begin{array}{l}\text { Cavities closed, } \\
\text { sputum negative, } \\
\text { discharged }\end{array}$ & $\begin{array}{l}\text { Before op. } 84 \\
\text { After 2nd stage } 82\end{array}$ & $\begin{array}{l}83 \\
78\end{array}$ & $\begin{array}{l}10 \\
428\end{array}$ & $\begin{array}{l}88 \\
91\end{array}$ & $\begin{array}{l}0.98 \\
0.95\end{array}$ & -2 & -5 \\
\hline 5 & H. K. & 22 & $\mathbf{M}$ & $\begin{array}{l}\text { Bilateral, advanced, } \\
\text { cavities R.U.L. }\end{array}$ & $\begin{array}{l}\text { Right, } 5 \text { ribs, lucite } \\
\text { balls, } 2 \text { stages }\end{array}$ & $\begin{array}{l}\text { Cavities closed, } \\
\text { spread R.L.I.., } \\
\text { sputum positive }\end{array}$ & $\begin{array}{ll}\text { Before op. } & 68 \\
\text { After 1st stage } & 61 \\
\text { After 2nd stage } & 49\end{array}$ & $\begin{array}{l}39 \\
34 \\
35\end{array}$ & $\begin{array}{l}3 \cdot 93 \\
5 \cdot 176 \\
4486\end{array}$ & $\begin{array}{l}87 \\
89 \\
89\end{array}$ & $\begin{array}{l}0.57 \\
0.56 \\
0.72\end{array}$ & -19 & -4 \\
\hline 6 & M. S. & 20 & $\mathbf{M}$ & $\begin{array}{l}\text { Bilateral, advanced, } \\
\text { cavities L.U.L. }\end{array}$ & $\begin{array}{l}\text { Left, } 6 \text { ribs, lucite } \\
\text { balls, } 2 \text { stages }\end{array}$ & $\begin{array}{l}\text { Cavities closed, } \\
\text { sputum negative, } \\
\text { discharged }\end{array}$ & $\begin{array}{l}\text { Before op. } 65 \\
\text { After 2nd stage } 62\end{array}$ & $\begin{array}{l}76 \\
50\end{array}$ & $\begin{array}{l}3 \cdot 76 \\
4 \cdot 21\end{array}$ & $\begin{array}{l}93 \\
90\end{array}$ & $\begin{array}{l}1 \cdot 12 \\
081\end{array}$ & -3 & -26 \\
\hline 7 & Y. H. & 47 & $\mathbf{M}$ & $\begin{array}{l}\text { Bilateral, far } \\
\text { advanced, cavities } \\
\text { R.U.L. }\end{array}$ & $\begin{array}{l}\text { Right, } 6 \text { ribs, lucite } \\
\text { balls, } 2 \text { stages }\end{array}$ & $\begin{array}{l}\text { Apical rest cavity, } \\
\text { sputum positive } \\
\text { (culture) }\end{array}$ & $\begin{array}{l}\text { Before op. } 74 \\
\text { After 2nd stage } 72\end{array}$ & $\begin{array}{l}46 \\
57\end{array}$ & $\begin{array}{r}10 \cdot 2 \\
483\end{array}$ & $\begin{array}{l}80 \\
92\end{array}$ & $\begin{array}{l}062 \\
0.79\end{array}$ & -2 & +11 \\
\hline 8 & M. S. & 22 & $\mathbf{M}$ & $\begin{array}{l}\text { Bilateral, advanced } \\
\text { tension cavity, } \\
\text { R.U.L. }\end{array}$ & $\begin{array}{l}\text { Right, } 5 \text { ribs, lucite } \\
\text { balls, } 2 \text { stages. } \\
\text { R.U. lobectomy }\end{array}$ & $\begin{array}{l}\text { Rest cavity R.U.L., } \\
\text { sputum positive } \\
\text { (culture) } \\
\text { Sputum negative, } \\
\text { discharged }\end{array}$ & $\begin{array}{l}\text { Before op. } 60 \\
\text { After 2nd stage } 68 \\
\text { After lobectomy } 66\end{array}$ & $\begin{array}{l}59 \\
77 \\
86\end{array}$ & $\begin{array}{l}9 \\
3 \cdot 30 \\
7 \cdot 55\end{array}$ & $\begin{array}{l}88 \\
90 \\
88\end{array}$ & $\begin{array}{l}1 \\
1 \cdot 13 \\
1 \cdot 3\end{array}$ & +8 & +18 \\
\hline 9 & I. $\mathbf{N}$. & 33 & $\mathbf{M}$ & $\begin{array}{l}\text { Bilateral, advanced, } \\
\text { cavities R.U.L. }\end{array}$ & $\begin{array}{l}\text { Right, } 6 \text { ribs, lucite } \\
\text { balls, } 1 \text { stage }\end{array}$ & $\begin{array}{l}\text { Cavities closed } \\
\text { sputum negative, } \\
\text { discharged }\end{array}$ & $\begin{array}{ll}\text { Before op. } & 76 \\
\text { After op. } & 67 \\
\end{array}$ & $\begin{array}{l}74 \\
78\end{array}$ & $\begin{array}{l}3 \cdot 3 \\
3 \cdot 9\end{array}$ & $\begin{array}{l}93 \\
93\end{array}$ & $\begin{array}{l}0.95 \\
1 \cdot 16\end{array}$ & -9 & $\overline{+4}$ \\
\hline 10 & E. G. & 27 & $\mathbf{M}$ & $\begin{array}{l}\text { Bilateral, advanced, } \\
\text { cavities L.U.L. }\end{array}$ & $\begin{array}{l}\text { Left, } 6 \text { ribs, lucite } \\
\text { balls, } 1 \text { stage }\end{array}$ & $\begin{array}{l}\text { Cavities closed, } \\
\text { sputum negative, } \\
\text { discharged }\end{array}$ & $\begin{array}{ll}\text { Before op. } & 69 \\
\text { After op. } & 58\end{array}$ & $\begin{array}{l}39 \\
43\end{array}$ & $\begin{array}{l}5 \cdot 52 \\
4 \cdot 14\end{array}$ & $\begin{array}{l}88 \\
92\end{array}$ & $\begin{array}{l}0.57 \\
0.74\end{array}$ & -11 & +4 \\
\hline 11 & B. R. & 30 & $\mathbf{F}$ & $\begin{array}{l}\text { Bilateral, far } \\
\text { advanced, cavities } \\
\text { L.U.L. }\end{array}$ & $\begin{array}{l}\text { Left, } 5 \text { ribs, lucite } \\
\text { balls, } 1 \text { stage }\end{array}$ & $\begin{array}{l}\text { Cavities closed. } \\
\text { sputum negative, } \\
\text { discharged }\end{array}$ & $\begin{array}{ll}\text { Before op. } & 80 \\
\text { After op. } & 93 \\
\end{array}$ & $\begin{array}{l}70 \\
96 \\
\end{array}$ & $\begin{array}{l}5 \cdot 58 \\
6 \cdot 21\end{array}$ & $\begin{array}{l}90 \\
92\end{array}$ & $\begin{array}{l}0.87 \\
1.03 \\
\end{array}$ & +13 & +26 \\
\hline 12 & S. G. & 35 & $\mathbf{F}$ & $\begin{array}{l}\text { Bilateral, far } \\
\text { advanced, cavities } \\
\text { R.U.L. and } \\
\text { R.L.L. }\end{array}$ & $\begin{array}{l}\text { Right, } 7 \text { ribs, } \\
\text { "polystan," } 1 \\
\text { stage }\end{array}$ & $\begin{array}{l}\text { Cavities closed, } \\
\text { sputum negative, } \\
\text { discharged }\end{array}$ & $\begin{array}{ll}\text { Before op. } & 30 \\
\text { After op. } & 32\end{array}$ & $\begin{array}{l}28 \\
25\end{array}$ & $\begin{array}{l}7 \\
3 \cdot 28\end{array}$ & $\begin{array}{l}74 \\
76\end{array}$ & $\begin{array}{l}0.93 \\
078\end{array}$ & +2 & -3 \\
\hline 13 & K. F. & 34 & $\mathbf{M}$ & $\begin{array}{l}\text { Bilateral, advanced, } \\
\text { cavities L.U.L. }\end{array}$ & $\begin{array}{l}\text { Left, } 6 \text { ribs, } \\
\text { "polystan," } 1 \\
\text { stage }\end{array}$ & $\begin{array}{l}\text { Cavities closed, } \\
\text { sputum negative, } \\
\text { discharged }\end{array}$ & $\begin{array}{ll}\text { Before op. } & 52 \\
\text { After op. } & 44 \\
\end{array}$ & $\begin{array}{l}23 \\
23\end{array}$ & $\begin{array}{l}7 \cdot 59 \\
483\end{array}$ & $\begin{array}{l}72 \\
83 \\
\end{array}$ & $\begin{array}{l}0.44 \\
0.52 \\
\end{array}$ & -8 & $\overline{0}$ \\
\hline 14 & M.R. & 28 & $\mathbf{F}$ & $\begin{array}{l}\text { Bilateral, far } \\
\text { advanced, cavities } \\
\text { L.U.L. and } \\
\text { R.U.L. }\end{array}$ & $\begin{array}{l}\text { Left, } 6 \text { ribs, } \\
\text { “polystan," } 1 \\
\text { stage } \\
\text { Right, } 5 \text { ribs, } \\
\text { "polystan," 1 } \\
\text { stage }\end{array}$ & $\begin{array}{l}\text { Cavities closed, } \\
\text { sputum positive } \\
\text { Cavities closed, } \\
\text { sputum negative, } \\
\text { discharged }\end{array}$ & $\begin{array}{lr}\text { Before 1st op. } & 61 \\
\text { After 1st op. } & 61 \\
\text { After 2nd op. } & 52\end{array}$ & $\begin{array}{l}25 \\
39 \\
30\end{array}$ & $\begin{array}{l}2 \cdot 68 \\
5 \cdot 17 \\
4 \cdot 30\end{array}$ & $\begin{array}{l}81 \\
86 \\
85\end{array}$ & $\begin{array}{l}0.40 \\
0.64 \\
0.57\end{array}$ & $\begin{array}{r}0 \\
-9\end{array}$ & $\begin{array}{r}+14 \\
+5\end{array}$ \\
\hline 15 & I. B. & 52 & $\mathbf{M}$ & $\begin{array}{l}\text { Bilateral, far } \\
\text { advanced, cavities } \\
\text { L.U.L. and } \\
\text { R.U.L. }\end{array}$ & $\begin{array}{l}\text { Left, } 5 \text { ribs, } \\
\text { "polystan," } 1 \\
\text { stage } \\
\text { Right, } 5 \text { ribs, } \\
\text { "polystan," } 1 \\
\text { stage }\end{array}$ & $\begin{array}{l}\text { Cavities closed, } \\
\text { sputum positive } \\
\text { Cavities closed, } \\
\text { sputum negative }\end{array}$ & $\begin{array}{ll}\text { Before op. } & 69 \\
\text { After 1st op. } & 65 \\
\text { After 2nd op. } & 62\end{array}$ & $\begin{array}{l}37 \\
36 \\
33\end{array}$ & $\begin{array}{l}4 \cdot 8 \\
4 \cdot 40 \\
9 \cdot 16\end{array}$ & $\begin{array}{l}78 \\
80 \\
74\end{array}$ & $\begin{array}{l}0.54 \\
0.55 \\
0.54\end{array}$ & $\begin{array}{l}-4 \\
-7\end{array}$ & $\begin{array}{l}-1 \\
-4\end{array}$ \\
\hline 16 & R. L. & 32 & $\mathbf{F}$ & $\begin{array}{l}\text { Bilateral, far } \\
\text { advanced, cavities } \\
\text { L.U.L. and } \\
\text { R.U.L. }\end{array}$ & $\begin{array}{l}\text { Left, } 6 \text { ribs, } \\
\text { "polystan," } 1 \\
\text { stage } \\
\text { Right, } 5 \text { ribs, } \\
\text { " polystan," } 1 \\
\text { stage }\end{array}$ & $\begin{array}{l}\text { Cavities closed, } \\
\text { sputum positive } \\
\text { Cavities closed, } \\
\text { sputum negative }\end{array}$ & $\begin{array}{ll}\text { Before op. } & 68 \\
\text { After 1st op. } & 54 \\
\text { After 2nd op. } & 57\end{array}$ & $\begin{array}{l}59 \\
41 \\
53\end{array}$ & $\begin{array}{l}3.22 \\
3 \cdot 92 \\
5 \cdot 17\end{array}$ & $\begin{array}{l}91 \\
82 \\
89\end{array}$ & $\begin{array}{l}087 \\
0.76 \\
0.93\end{array}$ & $\begin{array}{l}-14 \\
-11\end{array}$ & $\begin{array}{r}-18 \\
-6\end{array}$ \\
\hline 17 & M.S. & 24 & $\mathbf{F}$ & $\begin{array}{l}\text { Bilateral, far } \\
\text { advanced, cavities } \\
\text { L.U.L. }\end{array}$ & $\begin{array}{l}\text { Left, } 6 \text { ribs, } \\
\text { "polystan," } 1 \\
\text { stage }\end{array}$ & $\begin{array}{c}\text { Rest cavity L.U.L., } \\
\text { sputum positive }\end{array}$ & $\begin{array}{lr}\text { Before op. } & 45.5 \\
\text { After op. } & 47\end{array}$ & $\begin{array}{l}60 \\
68\end{array}$ & $\begin{array}{l}16 \\
7 \cdot 24\end{array}$ & $\begin{array}{l}68 \\
83\end{array}$ & $\begin{array}{l}1 \cdot 3 \\
1 \cdot 45\end{array}$ & +2.5 & +8 \\
\hline
\end{tabular}




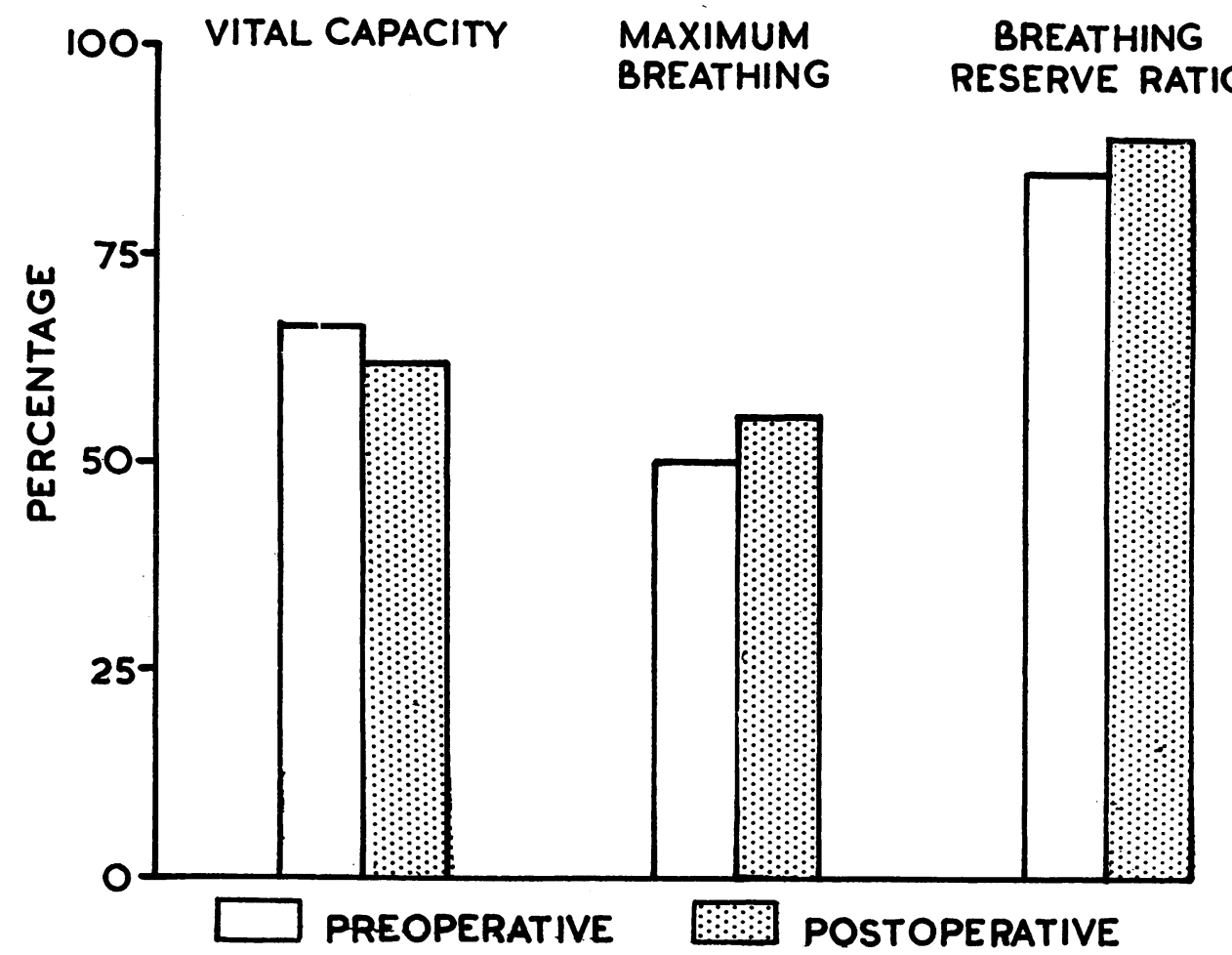

FIG. 1.-Avarage of vital capacity, maximum breathing capacity, and breathing reserve ratio in 17 patients before and after extraperiosteal plombage.

This shows that pulmonary function in all 17 patients was markedly impaired before the plombage procedure was undertaken.

The post-operative change of vital capacity ranged from -19 to $+13 \%$, the average being a loss of $4.7 \%$. The post-operative changes in maximum breathing capacity varied between -18 and $+33 \%$, the average being an increase of $6 \%$. The post-operative ventilation showed a tendency to decrease, i.e., a return towards normal values. The post-operative breathing reserve ratio showed an average of $87.9 \%$, indicating a slight improvement compared with the pre-operative level. The postoperative air velocity index showed a tendency to become normal in 11 cases, remained unchanged in two cases, and a further deviation was observed in four.

Fig. 1 is a diagrammatic comparison of pre- and post-operative average values of vital capacity, maximum breathing capacity, and breathing reserve ratio.

\section{COMMENT}

The results of our examinations show that following extraperiosteal plombage only a slight decrease of vital capacity occurred, whereas maximum breathing capacity even improved. Pathological hyperventilation tended to return towards normal in the majority of cases. The breathing reserve ratio showed a slight improvement and the air velocity index approached a more normal value (1.0) after operation.

The insignificant reduction of vital capacity after extraperiosteal plombage indicates that this procedure, by avoiding undesirable over-collapse, does not encroach on relatively normal pulmonary tissue. The slight improvement of maximum breathing capacity post-operatively tends to show that elasticity and distensibility of sound lung parenchyma are not interfered with by this procedure.

Watson and Gaensler (1952) found after extraperiosteal plombage an average vital capacity loss of $17 \%$ and an average maximum breathing capacity loss of $6 \%$. Comparing the pre-operative vital capacity and maximum breathing capacity values of their patients with those of ours, it is evident that pre-operative ventila'ory function was more severely impaired in our patients. The average vital capacity loss of $4.72 \%$ and the aver- 
age increase of $6 \%$ in maximum breathing capacity observed post-operatively in our patients compare favourably with Watson and Gaensler's results.

The effect of classical thoracoplasty on ventilatory function has been studied by many workers (Alexander, 1936, cited by Donaldson, 1947 ; Cournand and Richards, cited by Hochberg, 1952 ; Lambert, Berry, Cournand, and Richards, 1938 ; Leiner, 1946 ; Cournand and Richards, 1941), who report a vital capacity reduction varying between 20 and $50 \%$ and maximum breathing capacity reduction of 14 to $20 \%$. This is in consonance with our own observations in the two-stage seventh-rib thoracoplasty where average vital capacity losses of $20 \%$ and average maximum breathing capacity losses of $19 \%$ were found.

The considerable loss of function following classical thoracoplasty appears to be due to deformation of the chest wall by decostalization, particularly of anterior rib segments, rotoscoliosis, as well as undesirable collapse of sound lung tissue. Most of these shortcomings are avoided in extraperiosteal plombage by which little or no chest wall deformity or scoliosis are produced and where immediate and selective collapse is obtained in a one-stage operation. In our patient extraperiosteal plombage was resorted to, as this appeared to be the only permanent collapse procedure they could withstand without undue operative risk and avoiding additional severe impairment of ventilatory function.

\section{SUMMARY}

Pre- and post-operative ventilatory function tests were performed in 17 patients undergoing extraperiosteal plombage.

All patients had a significantly reduced ventila tory function before operation. Post-operatively, vital capacity decreased slightly, whereas maximum breathing capacity, ventilation, and breathing reserve rat:o improved.

We are grateful to Dr. K. Braun (Head of the Cardiovascular Research Laboratory) for his help and advice in the preparation of this article.

\section{REFERENCES}

Alexander, J. (1936). Ann. Surg., 104, 545. Cited by Donaldson. (1937). The Collapse Therapy of Pulmonary Tuberculosis. Thomas, Springfield, Illinois.

Baldwin, E. de F., Cournand, A., and R:chards, D. W., Jr. (1948) Medicine, Baltimore, 27, 243.

Birath, G. (1944). Acta med. scand., Suppl. 154.

Cournand, A., and Richards, D. W., Jr. (1941). Amer. Rev. Tuberc. 44, 123 .

Donaldson, J. K. (1947). Surgical Disorders of the Chest, 2nd ed. p. 408. Lea and Febiger, Philadelphia.

Engberg, H., and Hansen, J. L. (1953). Acta tuberc. scand., $28,45$.

Gaensler, E. A. (1950). Amer. Rev. Tuberc., 62, 17.

Hochberg, L. A. (1952). The Thoracic Surgical Patient, 1st ed.. p. 25. Grune and Stratton, New York.

Kaltreider, N. L., Fray, W. W., and Phillips, E. W. (1938). J. thorac. Surg., 7, 262.

Lambert, A. van S., Berry. F. B., Cournand, A., and Richards, D. W. Jr. (1938). Ibid., 7, 302.

I e.ner, G. C. (1946). Amer. Rev. Tuberc., 53, 195.

Powers, S. R., Jr., and Himmelstein, A. (1951). J.thorac. Surg., 22, 45. Watson, T. R., Jr., and Gaensler, E. A. (1952). Trans. nat. Tuberc. Ass., N.Y., 48, 288.

Wilson, D. A. (1946). Surg. Clin. N. Amer., 26, 1060.

Woods, F. M., and Buente, L. (1953). Amer. Rev. Tuberc., E8, 902. - Walker, J. H., and Schmidt, I. (1950). Dis. Chest, 18, 401. 